\title{
Numerical analysis of initiation of gigantic jets connecting thunderclouds to the ionosphere
}

\author{
Lizhu Tong $^{1}$, Kenichi Nanbu ${ }^{1}$, and Hiroshi Fukunishi ${ }^{2}$ \\ ${ }^{1}$ Institute of Fluid Science, Tohoku University, Sendai 980-8577, Japan \\ ${ }^{2}$ Department of Geophysics, Tohoku University, Sendai 980-8578, Japan \\ (Received May 17, 2004; Revised September 23, 2004; Accepted September 23, 2004)
}

\begin{abstract}
The initiation of giant electrical discharges called as "gigantic jets" connecting thunderclouds to the ionosphere is investigated by numerical simulation method in this paper. Using similarity relations, the triggering conditions of streamer formation in laboratory situations are extended to form a criterion of initiation of gigantic jets. The energy source causing a gigantic jet is considered due to the quasi-electrostatic field generated by thunderclouds. The quasi-electrostatic field is assumed to be axisymmetrical. We calculate the electric fields for different thundercloud charges. The electron dynamics from ionization threshold to streamer initiation are simulated by the Monte Carlo technique. It is found that gigantic jets are initiated at a height of $\sim 18-24 \mathrm{~km}$. This is in agreement with the observations. The distributions of electron positions and electron energies at different initiation heights are presented. The method presented in this paper could be also applied to the analysis of the initiation of other discharges such as blue jets and red sprites.
\end{abstract}

Key words: Electrical discharge, gigantic jet, thundercloud, ionosphere, electron energy distribution.

\section{Introduction}

The lightning from thundercloud toward the ground has been widely studied. It has been found, however, that more elusive forms, dubbed elves, sprites with and without sprite halos, and blue jets, flit above the thunderclouds. The elves appear at the heights of $\sim 70-90 \mathrm{~km}$ and extend over several $100 \mathrm{~km}$ horizontally (Fukunishi et al., 1996, 1997; Barrington-Leigh and Inan, 2001; Takahashi et al., 2003). They are visible for less than $0.1 \mathrm{~ms}$. Red sprites were found at the heights of $\sim 55-80 \mathrm{~km}$ with narrow tendrils extending below $55 \mathrm{~km}$ (Sentman et al., 1995; Lyons, 1996; Takahashi et al., 2003). Their visible lifetime is from a few to some tens of ms. Sprite halos appear at the heights of $\sim 70-85$ $\mathrm{km}$ for a short time of $\sim 1-2 \mathrm{~ms}$, sometimes preceding the development of streamer structures of sprites (BarringtonLeigh and Inan, 2001; Miyasato et al., 2002, 2003). Blue jets propagate from cloud tops to a height of $\sim 40 \mathrm{~km}$ with a lifetime of $300 \mathrm{~ms}$ (Wescott et al., 1995; Rowland, 1998). Recently a new type of lightning, i.e., gigantic jets, was discovered, which linked the top of thunderclouds to the overlying charged atmosphere, known as the ionosphere. Pasko et al. (2002) reported a video recording of a blue jet propagating upwards from a thundercloud to an altitude of $\sim 70$ $\mathrm{km}$. The event went across the normal upper limit for blue jets and lower terminal height of sprites. More recently, Su et al. (2003) reported their observations of five gigantic jets that spanned the thundercloud top at $16 \mathrm{~km}$ and the ionosphere at an elevation of $90 \mathrm{~km}$ within half a second. The upper body of gigantic jets was very similar to sprites, but

Copy right (c) The Society of Geomagnetism and Earth, Planetary and Space Sciences (SGEPSS); The Seismological Society of Japan; The Volcanological Society of Japan; The Geodetic Society of Japan; The Japanese Society for Planetary Sciences; TERRAPUB their lower body resembled blue jets. Depending on the shape of their upper halves, these gigantic jets were classified as tree jets or carrot jets. These events are potentially important factors in the model of the earth's electrical and chemical environment. The investigation of these events will help us to understand their contribution to the global electrical circuit (Pasko, 2003).

Observations of gigantic jets are not enough to understand the factors causing the initiation of these events because no associated cloud-to-ground (CG) lightning discharges were detected in the underlying thunderstorm (Pasko et al., 2002; Su et al., 2003). The conditions triggering gigantic jets are unknown. Finding such conditions is the aim of the present work.

Pasko and George (2002) proposed a three-dimensional fractal modeling for blue jets and blue starters. Their results showed that blue jets and blue starters can be formed by a fast accumulation of 100-150 C of positive thundercloud charge distributed in a volume with effective radius of $3 \mathrm{~km}$ near the cloud top at $15 \mathrm{~km}$. They pointed out that streamers could be initiated by individual electron avalanches in strong fields exceeding the breakdown threshold or by initial sharp points creating localized field enhancements. Unfortunately, these discussions were not represented in their model. As a typical case in their model, the electrostatic field generated by a thundercloud charge of $120 \mathrm{C}$ at the height of $15 \mathrm{~km}$ is much lower than the breakdown threshold (Pasko and George, 2002, figure 1). The field value is only a little higher than the minimum field required to support the propagation of positive streamers within the region around the height of $18 \mathrm{~km}$. The initiation of these events is still underestimated.

Since the extremely-low-frequency (ELF) radio waves 
associated with gigantic jets were detected in these events and a negative cloud to ionosphere (NCI) discharge could lead to the generation of these ELF waves (Su et al., 2003), here we consider the initiation criteria for gigantic jets which are NCI discharges. The energy source causing gigantic jets is assumed to be due to the quasi-electrostatic field generated by thunderclouds. We calculate the axisymmetrical quasi-electrostatic fields for different thundercloud charges. The criterion of initiation of gigantic jets is proposed using the extension of the triggering conditions of streamer formation in laboratory situations. The estimated initiation heights are in agreement with the observations. We simulate electron dynamics from ionization threshold to streamer initiation and also give electron energy distributions at different initiation heights.

\section{Thundercloud Charge Producing a Gigantic Jet}

The charge structure of thunderclouds has been studied extensively for many years. By widespread observational evidence, National Research Council (1986) elaborated that the interior of the thundercloud contains a dipolar charge distribution consisting of positive charge in the upper part of the cloud and negative charge below the positive. Also, the upper positive charge attracts negative ions to top of the cloud to form a negative screening layer. MacGorman and Rust (1998) proposed a working hypothesis of the gross charge distribution of the thundercloud, i.e., a positive dipole (positive above negative charge) or a tripole structure with a small lower positive charge plus an upper negative screening layer. Furthermore, they indicated that the actual thundercloud charge distribution is more complex and more than four vertically stacked charges have often been inferred in some situations. Based on these structures, a positive streamer could be initiated if the electrostatic field above thundercloud exceeds the ionization threshold of air (Pasko et al., 1996; Pasko and George, 2002). However, the dipole or tripole charge structure is inconsistent with the observations of gigantic jets. Pasko et al. (2002) reported that they found an upward transport of negative charges within a blue jet. By analyzing the observational results, Su et al. (2003) further indicated that gigantic jets might be negative cloudto-ionosphere (NCI) discharges. These observations evidence that a negative charge distribution might govern the electric field initiating gigantic jet since upward propagating negative streamers effectively transport negative charge upward. One of possible explanations for the inconsistency is that the negative charge region was possibly elevated in a tornadic storm (MacGorman and Rust, 1998, p. 240). There is, however, a larger possibility that an intracloud (IC) discharge or a positive cloud to ground $(+\mathrm{CG})$ discharge occurred during the charge accumulation of the thundercloud. Due to its weak high-frequency radiation signature in some situations, the event was possibly missed by the observational system (Pasko and George, 2002). It is known that IC discharge often occurs as a primarily vertical discharge between the negative and upper positive charge regions of the thundercloud (National Research Council, 1986, p. 92). The neutralization between the negative and upper positive charges causes the negative screening layer located at the top of the thundercloud to be a primary source to produce the strong electric field above thundercloud. The positive cloud to ground (+CG) discharge may cause the same effect. It creates a negative charge source consisted of the negative screening layer and the lower negative charge located within the dipole or tripole structure. In these circumstances, here we consider the electrostatic field above thundercloud generated by the thundercloud charge on the basis of a fast accumulation of a negative charge located at the top of thundercloud. We note that the charge distribution is quite simple but the domain in which our concern is above the thundercloud. Since no information about the distribution of thundercloud charge initiating gigantic jet is available and the charge distribution of thundercloud based on the known dipole or tripole structure is inconsistent with the observations of gigantic jets, it could be expected that the electrostatic field distribution above thundercloud, generated by the simplified charge distribution, would be equivalent to that by the actual charge distribution. The assumption can be improved as relative data on the subject become available.

The thundercloud charge dynamics can be mathematically represented by (Pasko et al., 1997)

$$
Q(t)=Q_{0} \frac{\tanh (t / \tau)}{\tanh (1)}, 0<t<\tau,
$$

where $Q_{0}$ is the magnitude of thundercloud charge and $\tau$ is the duration for accumulation of thundercloud charge. Pasko and George (2002) pointed out that the charge accumulation timescale can in some cases be very fast (fraction of a second). Here we assume the timescale to be $\sim 0.5-1$ s. It is noted that the functional variation tanh $(\cdot)$ in Eq. (1) is not critical for the physics of the phenomena modeled (Pasko et al., 1997).

The thundercloud charge is $Q(t)=$ $\int_{V} \rho_{-}(r, z, t) 2 \pi r d r d z$, where $V$ represents the whole computational domain. The charge density $\rho_{-}(r, z, t)$ is assumed to be a Gaussian spatial distribution given by $\rho_{-}(r, z, t)=\rho(t) e^{-\left[\left(z-z_{-}\right)^{2} / a^{2}+r^{2} / b^{2}\right]}, z_{-}$is the mean height of negative thundercloud charges and $\rho(t)$ is the charge density corresponding to $Q(t)$. In this work we set $z_{-}=16 \mathrm{~km}$ (Su et al., 2003), $a=2,4$, and $6 \mathrm{~km}$, and $b=2 \mathrm{~km}$. The charge $Q(t)$ for these three cases of $a$ are shown in Fig. 1 as cases 1, 2, and 3, where $t_{i g n 1}, t_{i g n 2}$, and $t_{i g n 3}$ represent the time of initiation of gigantic jets in accordance with three cases of $a=2,4$, and $6 \mathrm{~km}$.

The distribution of the absolute values of charge densities over height at $t=\tau$ corresponding to cases 1-3 is shown in Fig. 2, in which the dotted curve represents the charge density for $a=b=3 \mathrm{~km}$ with positive charge fixed at 10 $\mathrm{km}$ height that is commonly used in many sprite modeling studies (Pasko et al., 1997).

\section{Initiation Conditions of Gigantic Jet}

In order to generate a streamer in air there are three conditions to be fulfilled (Raizer et al., 1998). They are

1) The electric field must exceed the ionization threshold;

2) The initial plasma patch is sufficiently ionized so that the electric field generated by the space charges is 


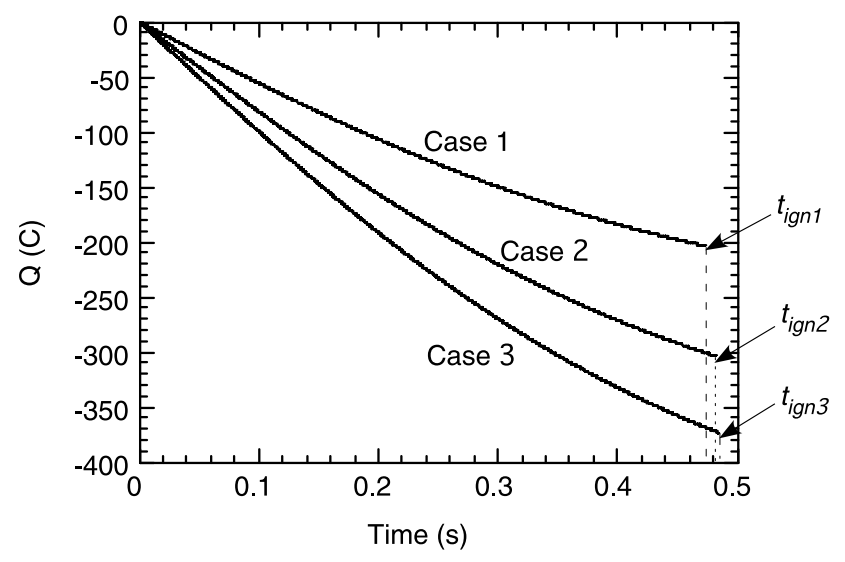

Fig. 1. Temporal evolution of thundercloud charges.

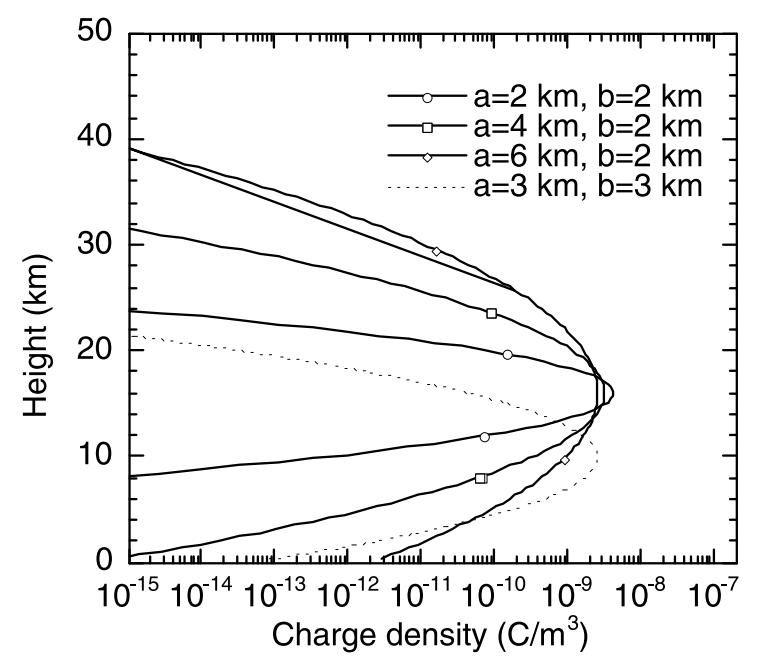

Fig. 2. Distribution of the absolute values of charge densities simulating thundercloud charges.

comparable with the external field itself;

3) A seed of free electrons is required to start the ionization.

A streamer is a self-adjusting object. It produces charged particles in a high field region at the front, needed to maintain high plasma conductivity in the channel. The electric field at streamer tip is controlled by the produced space charge. This property allows streamers to develop in weak fields, much lower than the ionization threshold of air. A minimum electric field strength is required to support stable streamer propagation. The minimum field corresponding to negative streamer propagation is $\sim 2-3$ times greater than that corresponding to the positive streamers (Raizer, 1991, p. 361; Babaeva and Naidis, 1997). Here we pay our attention on negative streamer propagation. The minimum field $-E_{c}$ required for propagation of negative streamers in air at atmospheric pressure is $\sim-12.5 \mathrm{kV} / \mathrm{cm}$ (Babaeva and Naidis, 1997), whose value has been used to study blue jets (Pasko and George, 2002). It is known that the electric field of $E_{0}=3.14 \times 10^{6} \mathrm{~V} \cdot \mathrm{m}^{-1}$ causes a breakdown of an air gap of $1 \mathrm{~cm}$ at atmospheric pressure and produces an effective ionization coefficient $\alpha_{\text {eff }}=1.24 \times 10^{3} \mathrm{~m}^{-1}$ (Raizer et al., 1998). The electric field for ionization threshold is estimated by $E_{k}=E_{0} \cdot\left(N / N_{0}\right)$, where $N_{0}=2.688 \times 10^{25} \mathrm{~m}^{-3}$ and $N$ is the number density of air molecules, taken from US Standard Atmosphere (1976).

The initiation of gigantic jets was detected to appear at the heights of $\sim 18-24 \mathrm{~km}$ (Su et al., 2003). The atmospheric pressure in that region is $\sim 50$ Torr. Under the pressure, the ordinary method estimating the interaction of charged particles with the external field, such as the Particle-in-Cell (PIC) method, requires a very fine mesh (e.g., less than $1 \mathrm{~mm}$ in $z$-direction). This will cause an intensive demand of CPU time even for modern computers. In this work, we use a simple method to estimate the interaction. We assume that the electric field inside a plasma patch generated by electrons and ions is equivalent to that caused by the electric dipole such that positive and negative charges locate at the centre of positive ions and electrons, respectively. The electric field in the middle of the electric dipole can be simply given by

$$
E_{d / 2}=\frac{q}{2 \pi \epsilon_{0}(d / 2)^{2}},
$$

where $d$ is the distance of electric dipole, $\epsilon_{0}$ is the permittivity of free space, and $q$ is the charge of the electric dipole. We note that the above method is rather simplified in comparison with the PIC method. However, as we discussed above, generating a streamer needs the growth of electric field inside the plasma patch up to the level compared with the external field. It is known that for the streamer corona, in spite of its internal structural complexity involving multiple highly branched streamer channels, its macroscopic characteristics remain relatively stable under a variety of external conditions. Therefore, we consider that a streamer is initiated if the electric field generated by electrons and ions makes the electric field inside the plasma patch reduce from $E_{k}$ to $E_{c} \cdot\left(N / N_{0}\right)$.

Following similarity relations, we obtain the minimum number $N_{\text {min }}$ of electrons generating a streamer in upper atmosphere by

$$
N_{\min }=8.68 \times 10^{7}\left(E_{0}-E_{c}\right) \cdot\left(N / N_{0}\right) \cdot d^{2} .
$$

The third condition to initiate a streamer can be naturally fulfilled in the domain close to the top of thundercloud if we consider gigantic jets which are negative cloud to ionosphere (NCI) discharges. The negative cloud may be regarded as a cathode that could emit electrons. In the previous theoretical modeling (e.g., QE model (Pasko et al., 1997)), the ambient electron number density is taken from observations, which is simplified as a function of height. However, these ambient electrons are usually considered within the domain over the height of $\sim 60-70 \mathrm{~km}$ (Cummer et al., 1998). In fact the gigantic jets are initiated below 30 $\mathrm{km}$ (Su et al., 2003), these ambient electron densities cannot be directly used for the study of gigantic jets. In the present work, we consider that electrons are emitted from the top of thunderclouds. Our calculation is terminated once the electron number exceeds $N_{\min }$. After that, a streamer will be initiated to form a gigantic get. 


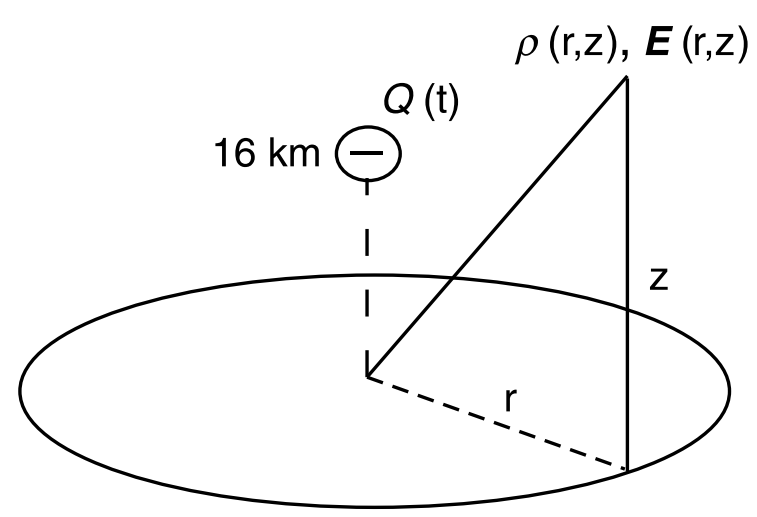

Fig. 3. Schematic diagram of thundercloud charge and coordinate system $(r, z)$.

\section{Quasi-electrostatic Field Generated by Thun- dercloud Charge}

The two-dimensional cylindrical coordinate system $(r, z)$ is used, where the $z$ axis represents the height. The schematic diagram is shown in Fig. 3. The computational domain is $r \leq 60 \mathrm{~km}$ and $0 \leq z \leq 90 \mathrm{~km}$. We assume that the quasi-electrostatic (QE) field is axisymmetrical. The three boundaries at $z=0 \mathrm{~km}, z=90 \mathrm{~km}$, and $r=60$ $\mathrm{km}$ are assumed to be perfectly conducting. The effect of the artificial boundary at $r=60 \mathrm{~km}$ on the QE field is considered as small (Pasko et al., 1997).

The continuity equation on the basis of charge conservation law is (Tong et al., 2004)

$$
\frac{\partial \rho}{\partial t}+\nabla \sigma \cdot \boldsymbol{E}+\rho \sigma / \epsilon_{0}=0,
$$

where $\rho$ is the charge density, $\sigma$ is the conductivity, and $t$ is the time. $\boldsymbol{E}$ is electrostatic field governed by

$$
\nabla \cdot \boldsymbol{E}=\left(\rho+\rho_{s}\right) / \epsilon_{0},
$$

where $\rho_{s}$ is the thundercloud source charge density, i.e., $\rho_{s}=\rho_{-}$in this work. Before the ionization threshold is reached, electron conductivity is low below $60 \mathrm{~km}$ height. Therefore the total conductivity $\sigma$ is dominated by ion conductivity, taken by $\sigma=5 \times 10^{-14} e^{z / 6} \mathrm{~km} S / \mathrm{m}$ (Dejnakarintra and Park, 1974). The ordinary finite difference method (Potter, 1973) is used to solve Eq. (4). Since $\boldsymbol{E}=-\nabla \phi$, where $\phi$ is the potential, Eq. (5) is transformed into: $\nabla^{2} \phi=$ $-\left(\rho+\rho_{s}\right) / \epsilon_{0}$. Considering the Dirichlet boundary conditions with respect to $z$ direction, the finite difference equation for $\phi$ is Fourier-sine transformed with respect to $z$ (Hockney and Eastwood, 1988; Kondo and Nanbu, 2001). We define the Fourier-sine transform

$$
\hat{\phi}_{i, n}=2 \sum_{j=1}^{N_{z}-1} \phi_{i, j} \sin \left(\pi n j / N_{z}\right),
$$

where $\hat{\phi}_{i, n}$ is the transform of $\phi_{i, j}\left[\equiv \phi\left(r_{i}, z_{j}\right)(i=\right.$ $\left.0,1, \ldots, N_{r} ; j=1,2, \ldots, N_{z}-1\right), i$ and $j$ are the variables of $r$ and $z$ directions, and $n$ is the transformed variable of $j$, and $N_{r}$ and $N_{z}$ are the number of cells in $r$ and $z$ directions, respectively. After the Fourier-sine transform, the finite difference equation of $\hat{\phi}_{i, n}$ becomes one-dimensional form

$$
B_{i} \hat{\phi}_{i-1, n}+D_{i, n} \hat{\phi}_{i, n}+A_{i} \hat{\phi}_{i+1, n}=C_{i, n} \text {, }
$$

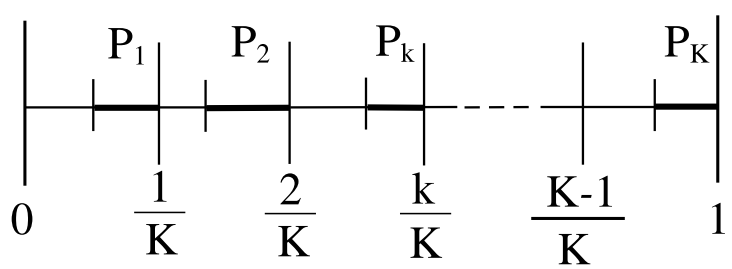

Fig. 4. The method to sample a collisional event.

Table 1. $Q(\mathrm{C}), H(\mathrm{~km}), t_{i o z}(\mathrm{~s})$ corresponding to ionization threshold.

\begin{tabular}{cccc}
\hline Cases & $Q$ & $H$ & $t_{i o z}$ \\
\hline Case 1 & 203.57 & 18.63 & 0.47 \\
\hline Case 2 & 303.63 & 20.74 & 0.48 \\
\hline Case 3 & 373.27 & 23.55 & 0.48 \\
\hline
\end{tabular}

where $i=1,2, \ldots, N_{r}-1$. The coefficients shown in Eq. (7) are

$B_{i}=\frac{2 r_{i-1 / 2}}{\Delta r_{i-1 / 2}}, \quad D_{i, n}=-\frac{2 r_{i+1 / 2}}{\Delta r_{i+1 / 2}}-\frac{2 r_{i-1 / 2}}{\Delta r_{i-1 / 2}}-\left(\frac{2}{\Delta z}\right)^{2}\left(\Delta r^{2}\right)_{i} \sin ^{2}\left(\frac{\pi n}{2 N_{z}}\right)$, $A_{i}=\frac{2 r_{i+1 / 2}}{\Delta r_{i+1 / 2}}, \quad C_{i, n}=-\frac{\hat{\rho}_{i, n}}{\epsilon_{0}}\left(\Delta r^{2}\right)_{i}$,

where $\hat{\rho}_{i, n}$ is the Fourier-sine transform of $\rho_{i, j}$, and we define

$\Delta r_{i+1 / 2}=r_{i+1}-r_{i}, \quad r_{i+1 / 2}=r_{i}+0.5 \times \Delta r_{i+1 / 2}$,

$\left(\Delta r^{2}\right)_{i}=r_{i+1 / 2}^{2}-r_{i-1 / 2}^{2}, \quad \Delta z_{j+1 / 2}=\Delta z_{j}=\Delta z$.

The method of Thomas algorithm (Hockney and Eastwood, 1988) are used in solving Eq. (7) and $\hat{\phi}_{i, n}$ is then inversely transformed to obtain $\phi_{i, j}$.

\section{Monte Carlo Method Simulating Electron Dy- namics}

The one-dimensional Monte Carlo simulation starts at the time that electric field arrives at ionization threshold. Several hundred, or thousand electrons are assumed as emitted from the top of thunderclouds. The equation of motion for the electrons is simulated by the modified Verlet method (Ueda, 1990)

$$
\begin{gathered}
z(t+\Delta t)=z(t)+v_{z}(t) \Delta t+F_{z}(t)(\Delta t)^{2} / 2 \mathrm{~m}, \\
v_{z}(t+\Delta t)=v_{z}(t)+\left[F_{z}(t+\Delta t)+F_{z}(t)\right] \Delta t / 2 \mathrm{~m},
\end{gathered}
$$

where $z$ and $v_{z}$ are the axial components of position and velocity of electrons, respectively, $m$ is electron mass, $\Delta t$ is time step, and $F_{z}$ is the force acting on electrons given by $F_{z}=q_{e} E_{z}$. Here $q_{e}$ is electronic charge and $E_{z}$ is the axial component of electric field. For a mixture of $\mathrm{N}_{2}$ and $\mathrm{O}_{2}$, we consider 33 types of electron-molecule collisions. In addition to elastic, rotational, and vibrational excitations, we consider electronic excitations of $\mathrm{N}_{2}\left(A^{3} \sum_{u}^{+}\right), \mathrm{N}_{2}\left(B^{3} \Pi_{g}\right)$, $\mathrm{N}_{2}\left(W^{3} \triangle_{u}\right), \quad \mathrm{N}_{2}\left(B^{\prime 3} \sum_{u}^{-}\right), \quad \mathrm{N}_{2}\left(a^{\prime 1} \sum_{u}^{-}\right), \quad \mathrm{N}_{2}\left(a^{1} \Pi_{g}\right)$, $\mathrm{N}_{2}\left(w^{1} \triangle_{u}\right), \quad \mathrm{N}_{2}\left(C^{3} \Pi_{u}\right), \quad \mathrm{N}_{2}\left(E^{3} \sum_{g}^{+}\right), \quad \mathrm{N}_{2}\left(a^{\prime \prime 1} \sum_{g}^{+}\right)$, and $13 \mathrm{eV}$, ionizations of $\mathrm{N}_{2}^{+}\left(X^{2} \sum_{g}^{+}\right), \mathrm{N}_{2}^{+}\left(A^{2} \Pi_{u}\right)$, and $\mathrm{N}_{2}^{+}\left(B^{2} \sum_{u}^{+}\right)$for $\mathrm{N}_{2}$, and electronic excitations of 

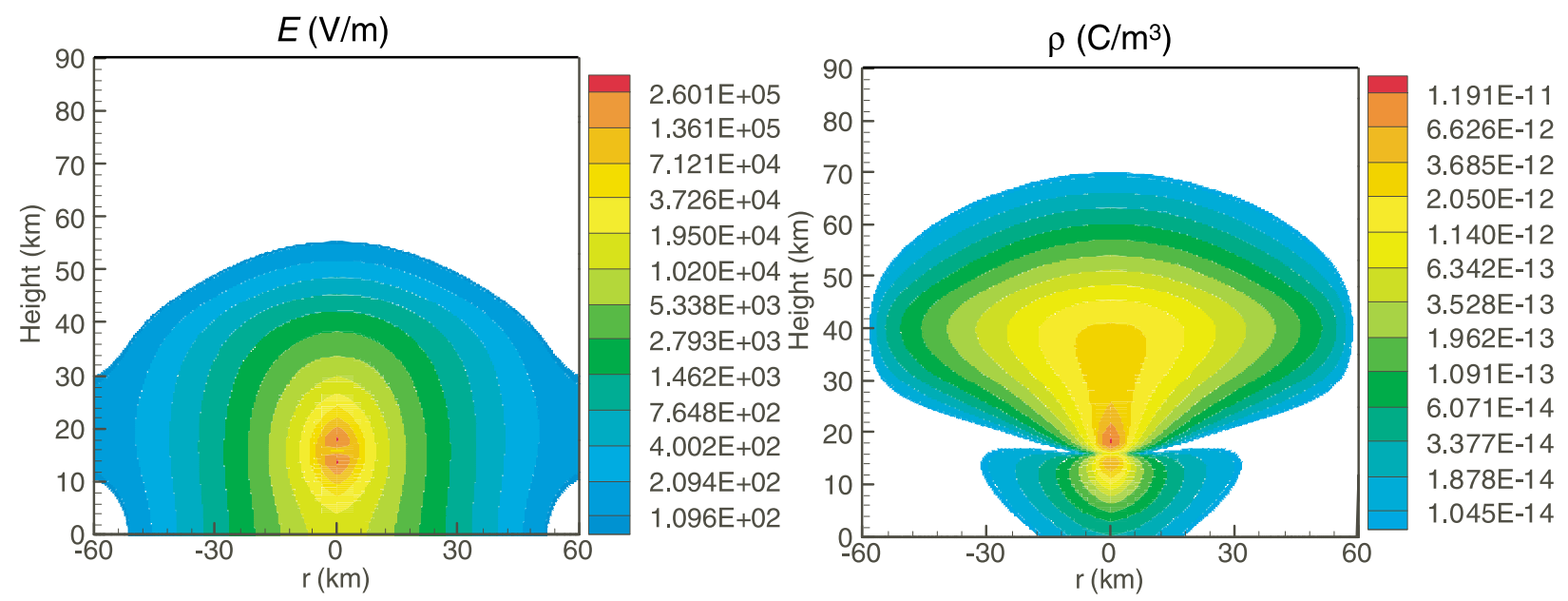

Fig. 5. Distribution of the absolute values of electric field $\boldsymbol{E}$ and charge density $\rho$ at the time of ionization threshold for case 1.

$\mathrm{O}_{2}\left(a^{1} \triangle_{g}\right), \mathrm{O}_{2}\left(b^{1} \sum_{g}^{+}\right), \mathrm{O}_{2}\left(c^{1} \sum_{u}^{-}\right), 6.0 \mathrm{eV}, 8.4 \mathrm{eV}$, and $10 \mathrm{eV}$, ionizations of $\mathrm{O}_{2}^{+}\left(X^{2} \Pi_{g}\right), \mathrm{O}_{2}^{+}\left(a^{4} \Pi_{u}\right), \mathrm{O}_{2}^{+}\left(A^{2} \Pi_{u}\right)$, $\mathrm{O}_{2}^{+}\left(b^{4} \sum_{g}^{-}\right)$, and $\mathrm{O}_{2}^{+}\left({ }^{4} \sum^{2},{ }^{2} \sum_{s}\right)$, dissociative attachment, and dissociative excitation for $\mathrm{O}_{2}$.

The corresponding cross sections for these reactions are taken from Rees (1989), Rapp and Briglia (1965), and Phelps (1985). For the estimation of electron-molecule collisions, Nanbu (1994) proposed a simple method to simultaneously determine whether an electron collides and which collisional event occurs in the case of collision.

By the method, we obtain the collision probability of the $i$ th electron for the $k$ th collisional type in $\Delta t$

$$
P_{k}=n_{n} \sigma_{k}\left(\varepsilon_{i}\right) v_{i} \Delta t, \quad(k=1,2, \ldots, K),
$$

where, $\varepsilon_{i}$ and $v_{i}$ are the energy and speed of the $i$ th electron, $\sigma_{k}\left(\varepsilon_{i}\right)$ is the cross section of the $i$ th electron for the $k$ th type collision, $n_{n}$ is the density of neutral gas, $\mathrm{N}_{2}$ or $\mathrm{O}_{2}$, and $K$ is the total number of collisional types, i.e., $K=33$ in the present work. The total probability that an electron collides with a molecule in $\Delta t$ is

$$
P_{T}=\sum_{k=1}^{K} P_{k}
$$

Equation (11) is written again as

$$
1=P_{T}+\left(1-P_{T}\right)=\sum_{k=1}^{K}\left[P_{k}+\left(\frac{1}{K}-P_{k}\right)\right] .
$$

Based on Eq. (12), the method to sample a collisional event is shown in Fig. 4. The unit length is divided into $K$ equal intervals and each interval is divided into two. We call a uniform random number $U(0<U<1)$. The integral part of $K U+1$ is the number of the $k$ th interval in which $U$ lies. The left part of the $k$ th interval is regarded as $1 / K-P_{k}$ and its right part is $P_{k}$. If $U$ lies in $P_{k}$, the $k$ th event occurs; otherwise the particle does not collide. Regarding the method for determining the post-collisional velocity of electron, please see Nanbu (2000).

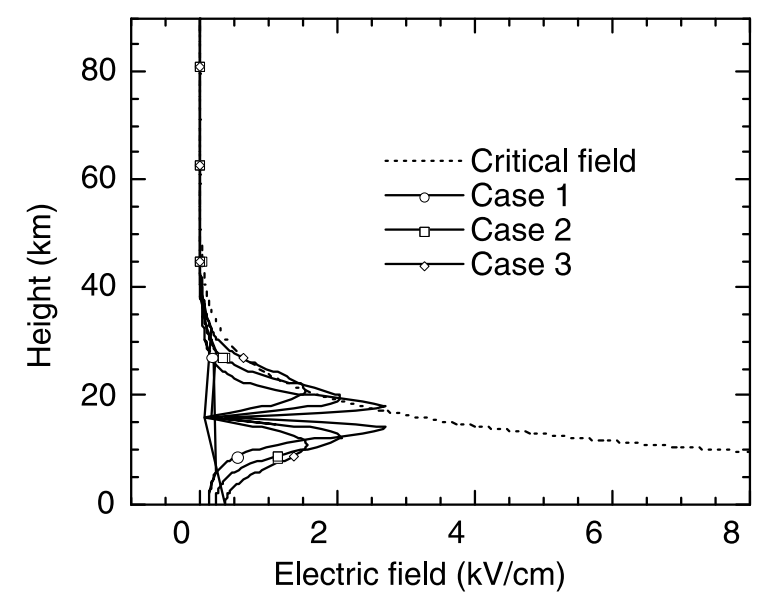

Fig. 6. Electric field on the axis $(r=0)$ compared with critical field $E_{k}$ for ionization.

\section{Results and Discussion \\ 6.1 Electric field and charge density}

We simulate the evolution of electric field and charge density during the accumulation of thundercloud charge for cases 1-3 defined in Fig. 1. Figure 5 shows the distribution of the absolute values of electric field and charge density at the time of ionization threshold for case 1 . The duration $\tau$ for accumulation of thundercloud charges is assumed to be $0.5 \mathrm{~s}$. Figure 6 gives the distribution of electric fields along $z$-axis for cases $1-3$. The electric field generated by thundercloud charges just exceeds the critical field $E_{k}$ for ionization. The first initiation condition of a gigantic jet is fulfilled. Table 1 gives the thundercloud charge $Q$, the height $H$ of ionization threshold, and the time $t_{i o z}$ corresponding to Fig. 6. $t_{i o z}$ is the duration from the accumulation of thundercloud charge to such a case that ionization threshold of air is reached, i.e., $E \geq E_{k}$ at $t=t_{i o z}$.

As seen in Table 1, the heights of $18.63-23.55 \mathrm{~km}$ for ionization threshold are in agreement with the observations of Su et al. (2003). They reported that an apparent emerging point of the gigantic jets started from the heights of $18 \mathrm{~km}$, $22 \mathrm{~km}$, and $24 \mathrm{~km}$. The thundercloud charges of 200-400 C have been used to study sprites and blue jets (Pasko et al., 


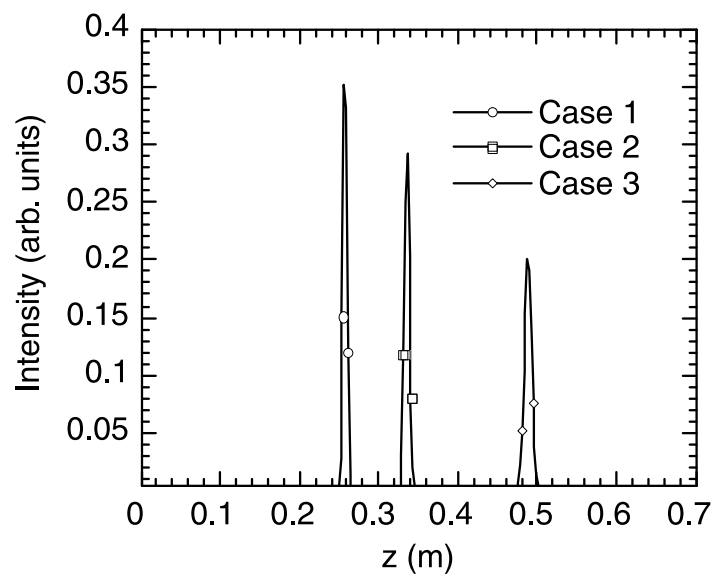

Fig. 7. The distribution of electrons started from the ionization heights at the time of initiation of gigantic jets.

1996, 1997). The magnitudes of charges shown in Table 1 could be reached by the accumulation of thundercloud.

6.2 Electron dynamics and electron energy distribution

In the Monte Carlo simulation we consider that the neutral gases are composed of $80 \% \mathrm{~N}_{2}$ and $20 \% \mathrm{O}_{2}$. We assume that about one thousand electrons are generated at the heights of ionization threshold. Then the electrons are accelerated and collide with neutral air molecules. The number of electrons is quickly increased due to ionizations. We follow electron dynamics until the electron number reaches $N_{\text {min }}$ given by Eq. (3). The time $t_{s t r}$ to initiate a streamer from ionization threshold is $1.94,2.53$, and $3.68 \mu$ s for cases $1-3$, respectively. The initiation time of gigantic jets is estimated as $t_{i g n}=t_{i o z}+t_{s t r}$. Figure 7 gives the distribution of electrons at the time of initiation of gigantic jets. The coordinate $z$ is counted from the ionization height $H$ shown in Table 1. For the ionization at a lower height, the motion of electrons is spatially more restricted due to high atmospheric pressure. With the increase of ionization height, electrons start to disperse, such is seen in case 3 in Fig. 7.

The electron energy distribution (EED) in upper atmosphere is attractive (Miyasato et al., 2003) because it allows us to analyze electron dynamics involving chemical reactions and the effect on upper atmosphere. In this work we examined the electron energy distribution. The electron energy distribution $E(\varepsilon)$ at the time of initiation of gigantic jets is given in Fig. 8. As seen in Fig. 8, the EEDs for cases 1-3 have a similar distribution, deviating from Maxwellian. The distribution has a low energy tail in which the energies of only a few electrons are over the ionization thresholds of gases, i.e., $15.58 \mathrm{eV}$ for $\mathrm{N}_{2}$ and $12.1 \mathrm{eV}$ for $\mathrm{O}_{2}$ (Tong et al., 2004). This is consistent with the electric field $\boldsymbol{E}$ generated by the thundercloud charge during the initiation of gigantic jets, which just exceeds the ionization threshold $E_{k}$ of air, as described in Section 6.1. The calculation shows that the largest electron energy appears around $\sim 16-$ $20 \mathrm{eV}$. The average electron energy is $\sim 4.97 \mathrm{eV}$, which is consistent with the previous research $(\sim 5 \mathrm{eV})$ of sprites (Pasko et al., 1997). In the present work we found that no runaway electrons appear. Moore et al. (2001) and Dwyer

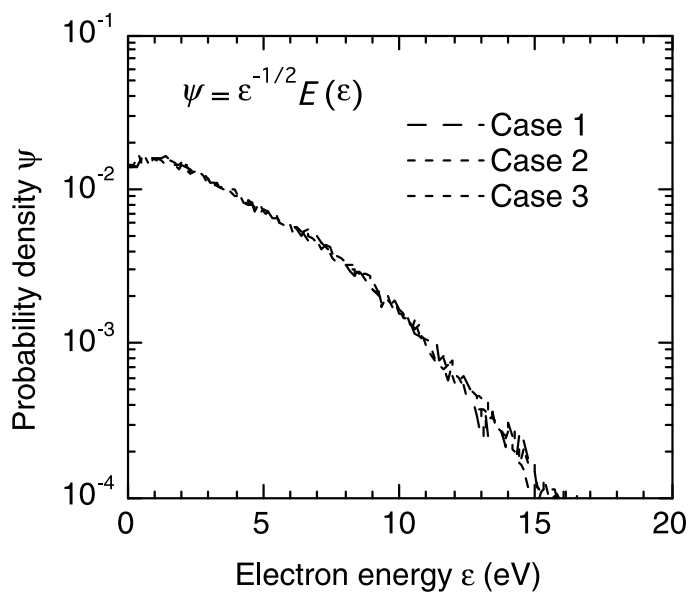

Fig. 8. Electron energy distribution at the time of initiation of gigantic jets.

et al. (2003) reported the radiation with the energies in excess of $1 \mathrm{MeV}$ associated with lightning leader discharges to the ground. They indicated that the runaway electrons may originate from the high energetic radiation. Thus, the runaway electrons could be expected to be generated after the transition from streamer coronas to leader discharges. Here, we focus our research on the initiation of gigantic jets with no association with CG lightning discharges. It is obvious that the runaway electrons are impossible to occur during the initiation of gigantic jets.

\section{Conclusion}

We investigate the initiation of gigantic jets connecting thunderclouds to the ionosphere by numerical simulation method. A criterion determining the initiation of gigantic jets is proposed on the basis of the triggering conditions of streamer formation in laboratory situations. Based on the present model, we found that the gigantic jets appear at 18-24 km height, which is consistent with the observations. The electron energy distribution during the initiation of gigantic jets deviates from Maxwellian distribution. The average electron energy is $\sim 4.97 \mathrm{eV}$ and the largest electron energy reaches $\sim 16-20 \mathrm{eV}$.

The present model presents the first step in studying gigantic jets. In reality, the gigantic jets possess a number of branches generated by different charged plasma channels. Studies of multiple streamers in the upper atmosphere is a much more complicated problem, which will be our next aim. These studies will make us have a better understanding for gigantic jets, a new giant electrical discharge phenomenon. This is not only interesting for basic science, but also important for solving aeronautics problems in the future.

Acknowledgments. The vector computer SX5 at the Institute of Fluid Science of Tohoku University is used in the present simulation. We would like to thank Dr. Dirk Lummerzheim for providing us the data of cross sections for electron-molecule collisions. 


\section{References}

Babaeva, N. Yu. and G. V. Naidis, Dynamics of positive and negative streamers in air in weak uniform electric fields, IEEE Trans. Plasma Sci., 25, 375-379, 1997.

Barrington-Leigh, C. P. and U. S. Inan, Identification of sprites and elves with intensified video and broadband array photometry, J. Geophys. Res., 106, 1741-1750, 2001.

Cummer, S. A., U. S. Inan, and T. F. Bell, Ionospheric D region remote sensing using VLF radio atmospherics, Radio Sci., 33, 1781-1792, 1998.

Dejnakarintra, M. and C. G. Park, Lightning-induced electric fields in the ionosphere, J. Geophys. Res., 79, 1903-1910, 1974.

Dwyer, J. R., M. A. Uman, H. K. Rassoul, M. Al-Dayeh, L. Caraway, J. Jerauld, V. A. Rakov, D. M. Jordan, K. J. Rambo, V. Corbin, and B. Wright, Energetic radiation produced during rocket-triggered lightning, Science, 299, 694-696, 2003.

Fukunishi, H., Y. Takahashi, M. Kubota, and K. Sakanoi, Elves: lightninginduced transient luminous events in the lower ionosphere, Geophys. Res. Lett., 23, 2157-2160, 1996.

Fukunishi, H., Y. Takahashi, M. Sato, A. Shono, M. Fujito, and Y. Watanabe, Ground-based observations of ULF transients excited by strong lightning discharges producing elves and sprites, Geophys. Res. Lett., 24, 2973-2976, 1997.

Hockney, R. W. and J. W. Eastwood, Computer Simulation using Particles, IOP Publishing Ltd, Bristol, 1988.

Kondo, S. and K. Nanbu, Axisymmetrical particle-in-cell/Monte Carlo simulation of narrow gap plane magnetron plasmas. I. Direct currentdriven discharge, J. Vac. Sci. Technol. A., 19, 830-837, 2001.

Lyons, W. A., Sprites observations above the US High Plains in relation to their parent thunderstorm systems, J. Geophys. Res., 101, 29,64129,652, 1996.

MacGorman, D. R. and W. D. Rust, The Electrical Nature of Storms, Oxford University Press, 1998.

Miyasato, R., M. J. Taylor, H. Fukunishi, and H. C. StenbaekNielsen, Statistical characteristics of sprite halo events using coincident photometric and imaging data, Geophys. Res. Lett., 29, 2033, doi:10.1029/2001GL014480, 2002.

Miyasato, R., H. Fukunishi, Y. Takahashi, M. J. Taylor, Energy estimation of electrons producing sprite halos using array photometer data, $J$. Atmos. Terr. Phys., 65, 573-581, 2003.

Moore, C. B., K. B. Eack, G. D. Aulich, and W. Rison, Energetic radiation associated with lightning stepped-leaders, Geophys. Res. Lett., 28, 2141-2144, 2001.

Nanbu, K., Simple method to determine collisional event in Monte Carlo simulation of electron-molecule collision, Jpn. J. Appl. Phys., 33, 47524753, 1994.

Nanbu, K., Probability theory of electron-molecule, ion-molecule, molecule-molecule, and Coulomb collisions for particle modeling of materials processing plasmas and gases, IEEE Trans. Plasma Sci., 28, 971-990, 2000.

National Research Council, The Earth's Electrical Environment, National
Academy Press, 1986.

Pasko, V. P., Electric jets, Nature, 423, 927-929, 2003.

Pasko, V. P. and J. J. George, Three-dimensional modeling of blue jet and blue starters, J. Geophys. Res., 107, 1458, doi:1029/2002JA009473, 2002.

Pasko, V. P., U. S. Inan, and T. F. Bell, Blue jets produced by quasielectrostatic pre-discharge thundercloud fields, Geophys. Res. Lett., 23, 301-304, 1996.

Pasko, V. P., U. S. Inan, T. F. Bell, and Y. N. Taranenko, Sprites produced by quasi-electrostatic heating and ionization in the lower ionosphere, $J$. Geophys. Res., 102, 4529-4561, 1997.

Pasko, V. P., M. A. Stanley, J. D. Mathews, U. S. Inan, and T. G. Wood, Electrical discharge from a thundercloud top to the lower ionosphere, Nature, 416, 152-154, 2002.

Phelps, A. V., JILA Information Center Report No. 26, University of Colorado, Boulder CO, 1985.

Potter, D., Computational Physics, John Wiley, New York, 1973.

Raizer, Yu. P., Gas Discharge Physics, Springer-Verlag, Berlin, 1991.

Raizer, Yu. P., G. M. Milikh, M. N. Shneider, and S. V. Novakovski, Long streamers in the upper atmposphere above thundercloud, J. Phys. D: Appl. Phys., 31, 3255-3264, 1998.

Rapp, D. and D. D. Briglia, Total cross sections for ionization and attachment in gases by electron impact. II. Negative-ion formation, J. Chem. Phys., 43, 1480-1489, 1965.

Rees, M. H., Physics and Chemistry of the Upper Atmosphere, Cambridge University Press, New York, 1989.

Rowland, H. L., Theories and simulations of elves, sprites and blue jets, $J$. Atmos. Terr. Phys., 60, 831-844, 1998.

Sentman, D. D., E. M. Wescott, D. L. Osborne, D. L. Hampton, and M. J. Heavener, Preliminary results from the Sprites94 aircraft campaign, 1, Red sprites, Geophys. Res. Lett., 22, 1205-1208, 1995.

Su, H. T., R. R. Hsu, A. B. Chen, Y. C. Wang, W. S. Hsiao, W. C. Lai, L. C. Lee, M. Sato, and H. Fukunishi, Gigantic jets between a thundercloud and the ionosphere, Nature, 423, 974-976, 2003.

Takahashi, Y., R. Miyasato, T. Adachi, K. Adachi, M. Sera, A. Uchida, and H. Fukunishi, Activities of sprites and elves in the winter season, Japan, J. Atmos. Solar. Terr. Phys., 65, 551-560, 2003.

Tong, L., K. Nanbu, Y. Hiraki, and H. Fukunishi, Particle modeling of the electrical discharge in the upper atmosphere above thundercloud, $J$. Phys. Soc. Jpn., 73(9), 2438-2443, 2004.

Ueda, A., Computer Simulation-Atomic Motion in Macro-system, Asakura Bookstore Publishing Ltd, Tokyo, 1990 (in Japanese).

US Standard Atmosphere 1976, NOAA-S/T 76-1562, US Government Printing Office, Washington, DC, 1976.

Wescott, E. M., D. D. Sentman, D. L. Osborne, D. L. Hampton, and M. J. Heavner, Preliminary results from the Sprites 94 aircraft campaign, 2, Blue jets, Geophys. Res. Lett., 22, 1209-1212, 1995.

L. Tong (e-mail: tong@ifs.tohoku.ac.jp), K. Nanbu, and H. Fukunishi 